\title{
Asthma phenotyping, therapy, and prevention: what can we learn from systems biology?
}

\author{
Alexandra Sittka', Julio Vera ${ }^{2}$, Xin Lai ${ }^{1,2}$ and Bernd T. Schmeck ${ }^{1,3}$
}

Asthma has a high prevalence worldwide, and contributes significantly to the socioeconomic burden. According to a classical paradigm, asthma symptoms are attributable to an allergic, Th2-driven airway inflammation that causes airway hyperresponsiveness and results in reversible airway obstruction. Diagnosis and therapy are based mainly on these pathophysiologic concepts. However, these have increasingly been challenged by findings of recent studies, and the frequently observed failure in controlling asthma symptoms. Important recent findings are the protective "farm effect" in children, the possible prenatal mechanisms of this protection, the recognition of many different asthma phenotypes in children and adults, and the partly disappointing clinical effects of new targeted therapeutic approaches. Systems biology approaches may lead to a more comprehensive view of asthma pathophysiology and a higher success rate of new therapies. Systems biology integrates clinical and experimental data by means of bioinformatics and mathematical modeling. In general, the "-omics" approach, and the "mathematical modeling" approach can be described. Recently, several consortia have been attempting to bring together clinical and molecular data from large asthma cohorts, using novel experimental setups, biostatistics, bioinformatics, and mathematical modeling. This "systems medicine" approach to asthma will help address the different asthma phenotypes with adequate therapy and possibly preventive strategies.

\section{INTRODUCTION}

Asthma is classically defined as an inflammatory chronic airway disease characterized by reversible airway obstruction and airway hyperresponsiveness (1). It affects 200-300 million people worldwide, and its prevalence has increased over the past few decades (2). Up to $10 \%$ of all cases of asthma are considered to be severe (3). Typically, the inflammation in asthma is described as being allergic, eosinophilic, IgE dependent, and Th2 driven (4). Therefore the therapy (besides bronchodilation) mainly addresses eosinophilic inflammation, either nonspecifically using topical or systemic glucocorticoids or antileukotriene drugs, or specifically addressing IgE using "biological therapies"; in the future, interleukin (IL)-13, IL-5, and others may also find use (5). However, in many patients it has not been found to be impossible to achieve sufficient control of asthma symptoms. This is partly because $>50 \%$ of asthma patients do not show a persistent eosinophilic inflammation (6), thereby seriously challenging the classical pathophysiological concepts. Systems-based approaches can help to establish a comprehensive and testable new hypothesis of the pathophysiology of the different phenotypes of asthma (7).

\section{CLINICAL CHALLENGES IN ASTHMA}

Caring for patients with asthma, especially children, provides many challenges, including long-term patient education, addressing socioeconomic and psychological issues, and choosing the right inhalation device,. In this paper we focus on some issues arising from recent pathophysiologic findings that can benefit from a systems biology or systems medicine approach.

\section{"Farm Effect"}

The risk and the disease course in asthma are associated with environmental factors. More than 20 years ago, it was proposed that "...allergic diseases are prevented by infection in early childhood, transmitted by unhygienic contact with older siblings, or acquired prenatally..." (8). In the same year, a report from Sweden (9) implicated a protective effect of rural environment for asthma or allergic rhinitis. In children of ages 5 to 7 years living on a farm in Germany, contact with livestock seemed to protect against asthma and atopy (10). In rural areas in Austria, Germany, and Switzerland, it has been shown that exposing children under the age of 1 year to stables and feeding them farm milk reduced the prevalence of asthma and, independently, atopic sensitization as well. This protective effect was not observed in children living in the same area who did not have direct contact with a farm environment. The protective effect was strongest in children who had been exposed to stables up to the age of 5 years (11). The effect was specifically correlated with exposure to cattle, pigs and poultry, hay, grain, straw, and silage, and the consumption of unprocessed cow's milk, thereby suggesting that the ingestion and/ or inhalation of certain substances in the farm environment are highly relevant to the protective effect (12). Other studies found that asthma was negatively or positively correlated with various infections (13). Colonization with Helicobacter pylori 


\section{Review | sittkaetal.}

was demonstrated to be negatively correlated with childhood asthma (14). In the same rural environment, a study tested the hypothesis that exposure to a particular microbial component (lipopolysaccharide) rather than infection per se might offer a protective effect against asthma (15). A later study has shown a protective effect against asthma for a constituent of peptidoglycan from Gram-negative or Gram-positive bacteria (16). Interestingly, prevalence of hay fever, atopic asthma, and atopic sensitization-but not nonatopic wheeze-in children was inversely related to endotoxin levels in samples of dust from the children's mattresses. More recently, the same group of researchers showed that the protective effect against asthma correlated with the diversity of microbial exposure, especially exposure to particular fungal and bacterial (Listeria monocytogenes, Bacillus licheniformis, and others) species (17).

It was possible to mimic these effects in murine models of allergic airway inflammation. In the classical approach, Th2biased mouse inbred strains were sensitized by intraperitoneal application of ovalbumin/aluminium hydroxide (as adjuvant) and challenged by ovalbumin inhalation to provoke eosinophilic airway inflammation. In primary preventive approaches, intranasal application of substances or nonpathogenic microbes found in cowshed dust, fodder, or farm children's mattresses was shown to reduce or prevent eosinophilic airway inflammation and airway hyperresponsiveness (12). Among other agents, arabinogalactanes, Gram-positive and Gram-negative bacteria, and dust extracts were tested.

A recent meta-analysis of published literature up to September 2011 analyzed data from 39 studies on the "farm effect" in asthma (18). Most of these studies applied cross-sectional approaches, and there were great variances and heterogeneity in definitions, in the prevalence of asthma or wheeze, as well as in the extent of reported correlations. Despite these limitations, childhood exposure to farming environment appears to account for a $25 \%$ lower prevalence of doctor-diagnosed asthma and current wheeze.

The heterogeneity in protective effects has been attributed to several factors. For instance, the assessment of a "farming environment exposure" generally relied on retrospective descriptions of places of upbringing, parents' occupation, etc. (18). In addition, it has been argued that the farming environment in Switzerland, Austria, and Southern Germany may be characterized specifically by small and traditional farms containing only livestock, in particular cattle, and involving close contact between the children and the farm environment (12). Also, the usefulness of "doctor-diagnosed asthma" may be hampered by variances in definitions of asthma and in disease labeling. In addition, the tendency to label a disease condition as "asthma" might be greater in some populations (19).

One argument that questions the classical hypothesis of the role of hygiene in asthma is based on some pathophysiologic observations. For example, the protective effect is studied mostly on an atopic, eosinophilic, and Th2-driven background, but cowshed dust components do not change the inflammatory environment toward the Th1 direction (12). In fact, a farm environment seems to offer protection against nonatopic asthma or wheeze as well $(11,20)$. Another reason for skepticism of the classical hypothesis is that the overall decline in asthma prevalence in children and adults over the past decade does not seem to correlate with decreased hygiene, and neither does the increase in asthma prevalence in US inner city populations correlate with increased hygiene (13). Ironically, adults in a farming environment might face an increased risk for nonatopic asthma (21). An interesting new idea in this context is the "diversity hypothesis" of atopy (22). It suggests that too low a level of contact with natural environmental biodiversity adversely affects the human commensal microbiota, leading to inadequate stimulation of immunoregulatory circuits. A recent study in 14- to 18-year-old children in eastern Finland revealed that atopic individuals had lower environmental biodiversity in the surrounding of their homes and significantly lower generic diversity of Gram-negative Gammaproteo bacteria on their skins (23). Taken together, the "farm effect" or a modified "hygiene hypothesis" may offer potential for a truly preventive strategy in asthma. However, recent observations show inconsistent results, and there is a strong urge for a more comprehensive picture. This can possibly be achieved by modern "omics" technologies using systems approaches (24).

\section{Prenatal Asthma Protection}

Various observations imply that there may be a trans-placental transmission of asthma risk from mother to child (25). A potentially preventive strategy could arise from an interesting offshoot of the "farm effect"; because atopy and wheeze can occur early in life, it is likely that the predisposing events happen even earlier. A study in a British cohort showed that the use of antibiotic drugs during pregnancy increased the newborn's risk for wheeze and asthma (26). This effect did not depend on the group of antibiotics to which the drug belonged or the trimester of pregnancy during which the drug was taken, but increased with the overall number of antibiotics administered. Ege et al. found that maternal exposure to a farming environment during pregnancy increased atopic sensitization of the offspring (27), suggesting a role for intrauterine and epigenetic mechanisms. A European cross-sectional questionnaire survey also showed that maternal exposure to a farm environment during pregnancy had a protective effect against asthma and hay fever in the offspring(28). In general, a very tight interaction has been shown to exist between maternal trans-placental signals and the fetal immune system; for instance, maternal cells cross the placental barrier, settle in the offspring's lymph nodes until early adulthood, and suppress anti-maternal immune reactions by induction of fetal regulatory T cells (29). A glimpse of the specific immunologic background of this in utero protective effect against asthma is seen in the results of an analysis of cord blood $\mathrm{T}$ cells from 82 mothers in a rural area of southern Germany (30). In the cord blood samples of children of mothers who had lived and worked regularly on a farm during pregnancy, the number and function of regulatory $\mathrm{T}$ cells were increased, and IL-5 secretion in response to innate stimulation was reduced. Following up on this, a prospective study in a multinational birth cohort 
demonstrated that farm exposure enhances the secretion of interferon- $\gamma$ (IFN $\gamma)$ and tumor necrosis factor- $\alpha$ in response to stimulation of cord blood mononuclear cells, whereas no differences could be observed within respect toof IL-5, IL-10, and IL-12 (31). Similarly, in an Australian prospective birth cohort, a negative correlation was observed between IL-4- and IFN $\gamma$-levels in cord blood and asthma at 6 years of age (32). In contrast, elevated tumor necrosis factor- $\alpha$ levels correlated negatively with atopy only, and not with asthma.

This in utero preventive effect was reproduced in a murine ovalbumin model of allergic airway inflammation. Exposure to lipopolysaccharide before conception and during pregnancy reduced eosinophilic airway inflammation in the offspring in response to challenge with ovalbumin (33). More specifically, female mice were exposed intranasally before conception to Acinetobacter lwoffi, a Gram-negative bacterium found in cowshed dust (34). The asthma-protective effect in the offspring depended on low-level maternal inflammation. This could be inhibited by maternal knockout of Toll-like receptors, thereby mediating bacterial recognition. An interesting mechanistic insight arose from a study using the same experimental model (35). The Th2-response in the offspring was found to be reduced by elevated levels of IFN $\gamma$. Maternal bacterial exposure prevented histone modifications at T-cell promoters of IFN $\gamma$ and IL-4, but not IL-5, after ovalbumin challenge. The protective bacterial effect mediated by increased histone $\mathrm{H} 4$ acetylation at the IFN $\gamma$ promoter was preventable by use of an inhibitor of histone acetyl transferases. So far, nothing is known about the epigenetic effects on other asthma-relevant promoters.

Taken together, prenatal asthma protection afforded by a farming environment might be mediated by trans-placental transfer of cytokines and subsequent epigenetic events, e.g., in fetal immune cells. Further studies are needed to draw a comprehensive picture of these events in animal models as well as in humans.

\section{Many Different Phenotypes?}

For a long time, asthma has been characterized clinically on the basis of intermittent respiratory symptoms, reversible airflow obstruction, nonspecific bronchial hyperresponsiveness, and airway inflammation. Motivated in part by the unsatisfactory effectiveness of treatment strategies, recent hypotheses suggest that asthma is a syndrome containing different phenotypes that could be characterized by environmental triggers or by immunologic or molecular markers (36).

In children, diagnosis and prognosis of asthma are complicated by the fact that wheezing and asthma in pre-schoolage children are very heterogeneous in clinical phenotypes. Although daily impairment is usually rare, short but recurrent infection-triggered exacerbations are associated with cough and wheeze. Nonatopic wheezing is especially challenging in terms of diagnosis, therapy, and prognosis.

Valuable parameters for diagnosis are partially reversible airflow obstruction and lung function, allergen sensitization, increased IgE levels, and blood eosinophilia. However, reliable lung function measurements cannot be achieved in very young children. In addition, there is as yet no specific parameter or biomarker predicting persistent asthma.

In terms of therapy, there are only limited data within respect toof different treatment regimes in young children, even for distinct phenotypes. The impact of the developing airways on drug deposition is an issue, as is the effect of inhaled corticosteroids on growth and development. Recent reports suggest that the daily use of inhaled corticosteroids in children is associated with a reduction in linear growth, and that symptom-driven inhalation of corticosteroids is equally effective with fewer side effects $(37,38)$. Overall, however, an effective strategy to prevent asthma or stop it from worsening is not yet in sight.

WithAs regard tos asthma in adults, the phenotype challenges remain. In a study aimed at phenotype identification by clinical features alone, data from 726 subjects from a persistent asthma cohort were analyzed using an unsupervised hierarchical cluster analysis of 34 clinical variables, including age at onset, gender, body weight, degree of airflow limitation, reversibility of airflow limitation, and frequency of asthma exacerbation (39). The authors showed that the resulting fivepatient cluster could be correctly characterized on the basis of onlymerely three clinical parameters: pre- and postbronchodilator percentage of predicted forced expiratory volume in $1 \mathrm{~s}$ and age of onset of asthma. However, this cluster did not correlate with the terms "severe asthma" and "treatment refractory asthma." In another study in 2,536 adults with asthma, latent class analysis was applied on 19 variables to identify four subgroups, including active treated allergic childhood-onset asthma and active treated adult-onset asthma (40). The groups partly correlated with eosinophil and neutrophil counts. A wider approach including clinical, physiologic, and pathologic parameters in a k-means cluster analysis has been performed by Haldar et al. (3). The authors studied two distinct asthma populations: a group from primary care with mild to moderate asthma and a group from secondary care with refractory asthma. In the mild asthma group, three clusters could be identified: (i) early-onset atopic asthma (airway dysfunction, asthma symptoms, and eosinophilic airway inflammation, frequent exacerbations requiring oral corticosteroids), (ii) obese noneosinophilic asthma (preponderance of female subjects, asthma symptoms, absence of eosinophilic airway inflammation), and (iii) benign asthma (middle-aged, little evidence of asthma symptoms, airway inflammation, airway hyperresponsiveness, and exacerbations). In the refractory asthma population, four clusters were identified: (i) early-onset atopic asthma (see earlier text), (ii) obese, noneosinophilic asthma (see earlier text), (iii) early-onset, symptom-predominant asthma (minimal eosinophilic disease), and (iv) eosinophilic inflammation-predominant asthma (few symptoms, late-onset disease, greater proportion of male subjects). The first two clusters were concordant; disease severity differed in cluster 1, which seemed to be strongly associated with a lack of patient compliance. Interestingly, degrees of eosinophilic inflammation and symptoms were discordant in clusters 2 and 3 of the 


\section{Review | sittkaetal.}

refractory asthma group. This may give rise to overtreatment and treatment failure with inhaled corticosteroids in these groups.

New phenotypes may be discovered even by small observational studies. For instance, a study in 19 subjects with severe asthma and requiring daily systemic corticoids applied a technique involving video-assisted thoracoscopic biopsies to identify a histologic phenotype of interstitial nonnecrotizing granulomas in 10 patients (41).

An important issue in defining subgroups of asthma in children or adults is the question of time, i.e., phenotype stability. Using repeated measures of sputum cytology in a cohort of 51 children with severe asthma and 28 with mild to moderate asthma, subjects were classified as eosinophilic, neutrophilic, mixed granulocytic, or paucigranulocytic. A total of $63 \%$ of the children were grouped into two or more phenotypes, and $41 \%$ were transiting between noneosinophilic and eosinophilic asthma (42). These changes do not correlate with inhaled corticosteroid treatment, but might depend on variable adherence to treatment protocols or to variations in exposure to allergens or viruses over time. Similarly, in adults with asthma who were not taking inhaled glucocorticoids, three subgroups could be defined by sputum cytology: persistent eosinophilia (22\%), intermittent eosinophilia (31\%), and persistent noneosinophilia (47\%). Not surprisingly, the third group did not benefit from a combined anti-inflammatory therapy with corticosteroids and a leukotriene receptor antagonist. Recent approaches aim to widen the field of phenotype definition from clinical presentation and sputum cytology to molecular markers and mechanisms (43).

The central goals of the phenotyping efforts are to devise improved and targeted therapeutical strategies, especially for difficult-to-treat asthma. In this context, the reduced effectiveness of inhaled glucocorticoids is an important challenge. It seems to be partly associated with a polymorphism in the glucocorticoid-induced transcript 1 gene (44). Even more prominent may be the effect of differences in phenotypes; patient groups with high symptom levels but low eosinophilic inflammation seem to have only moderate benefit from inhaled glucocorticoids (3). An issue in terms of therapy is also the retrospective observation that Americans with African ancestry showed greater deterioration when treated with long-acting $\beta$-agonists (45). There is a debate on whether treatment with this substance group alone without inhaled corticosteroids leads to increased mortality (46), and whether mutations in the $\beta 2$-adrenoreceptor have an impact on the drug's effect on peak flow and disease symptoms (47). In noneosinophilic subgroups of asthma patients, recent studies with low patient numbers suggest a beneficial effect of long-acting anticholinergic drugs (48), or macrolide antibiotics (49).

Considering all aspects together, there is a strong medical need and an intellectual rationale to not "abandon asthma as a disease concept" (50), but to undertake phenotyping or "endotyping" (51) even on the molecular and "omics" level (43) so as to improve clinical asthma therapy using targeted approaches.

\section{(Failure of) Targeted Therapies}

Given theThe fact that there are a significant number of patients with severe or difficult-to-treat asthma, andgiven the reluctance of patients to use glucocorticoids, there have been attempts to translate basic research findings into new clinical treatment strategies. Typically, they target a single factor of the assumed asthma pathogenesis.

Anti-IgE therapy has shown to reduce days with asthma symptoms, hospitalizations, exacerbations, and the daily dose of an inhaled glucocorticoid that was needed to maintain disease control in children (52). Responsive phenotypes seem to be characterized by higher blood eosinophil counts, higher levels of exhaled nitric oxide, a total IgE level of $>100 \mathrm{IU} / \mathrm{ml}$, and a body mass index of $>25$. Interestingly, sensitization and exposure to cockroach allergen was also predictive of a good clinical response.

The outcomes of anti-IL-5 therapy have been disappointing (53). With regard to (sub-) phenotypes, there are indications that anti-IL-5 therapy can lead to moderate improvement in lung function tests and reduced incidence of severe exacerbation in patients with uncontrolled, eosinophilic asthma $(54,55)$, and possibly nasal polyps (56).

Several strategies to block IL-4 signaling have been applied in asthma patients. Inhaled recombinant human sIL-4R showed initial promising effects on lung function and airway inflammation (57). However, results for this and other anti-IL-4 strategies have been inconsistent. Given that IL-4 and IL-13 are redundant in that they share the IL-4 receptor $\alpha$, strategies against this receptor have been developed, including a mutated recombinant human IL-4 ("IL-4 mutein"). First results of this approach hint at a beneficial effect in eosinophilic asthma (58).

In a cohort of 219 patients with poorly controlled asthma, anti-IL-13 therapy was shown to enhance the prebronchodilator forced expiratory volume in $1 \mathrm{~s}$ after $12 \mathrm{wk}$ of treatment, but did not reduce the occurrence of exacerbations and symptoms of asthma (59). The patient phenotype with the best treatment results seems to be characterized by higher levels of periostin (a cytokine produced by the lung epithelium in response to IL-13) and exhaled nitric oxide.

Challenging the most widely applied strategy to address modulators of the Th2 and IgE axis, data have emerged that support a role for tumor necrosis factor- $\alpha$ in human refractory asthma (60). However, recent studies targeting tumor necrosis factor- $\alpha$ in human subjects have failed to show improved clinical end points (61).

Most of these targeted therapeutical approaches have been initially successfully tested in mouse models. There are difficulties in translating these findings to the clinical setting because murine models have certain limitations: (i) mice are not humans, includingincluding in immunologic and pulmonary aspects (62), (ii) the classical experimental setup tests primary or secondary prevention rather than therapy, and (iii) the formerly common murine model used sensitization with ovalbumin and adjuvants such as aluminum hydroxide followed by a challenge with ovalbumin to provoke a Th2-driven eosinophilic airway inflammation and hyperresponsiveness (63). These 
models are certainly unphysiologic and, most importantly, tailored to mimic just one specific phenotype of human asthma. Therefore they might not be useful in predicting therapeutic success in a diverse patient population.

\section{SYSTEMS BIOLOGY WORKBENCH}

Systems biology is a new interdisciplinary approach to biomedical research. The key elements of this approach are: (i) the retrieval of large-scale sets of quantitative experimental data, either clinical or biological, and (ii) the use of advanced mathematical techniques to process, analyze, and interpret these data. The discoveries of the past decade have revealed that many diseases with high prevalence are of multi-factorial nature. It therefore becomes necessary to accumulate massive amounts of clinical and biological data in order to discover the origins of diseases and to improve success rates with respectas regards to early detection and treatment. Unfortunately, massive volumes of high-throughput biological data evade direct interpretation. Proteins, miRNAs, and metabolites are parts of large and complex biological regulatory networks, which often show counterintuitive behavior elicited by the complex nature of the interactions encoded in these networks. This calls for the use of powerful mathematical methods to dissect the data in order to find and interpret the complex relations hidden in them, explain the causes of diseases, and provide pointers about how to fight them.

Systems biology emerged as a strategy to tackle this difficult task of extracting new insights from complex quantitative biological data. To date, there is no standard for the application of systems biology to the investigation of biomedical problems; the strategies used vary depending on the nature and amount of data available and the questions to be addressed. We can distinguish two main approaches in systems biology — the "omics" approach with data analysis, and the mathematical modeling approach.

\section{The "Omics" Approach With Data Analysis}

This approach relies on the collection and analysis of massive volumes of clinical, quantitative, high-throughput data (genomic, transcriptomic, or proteomic), alone or in combination with other biometric information such as the prevalence rate of the investigated disease and other demographical data (Figure 1). The approach is used to analyze tissue- or plasma-specific concentrations of dozens to thousands of proteins, mRNAs, miRNAs, and other relevant biomolecules, extracted from a population sample relevant for the disease under investigation. Once collected and processed, the data are analyzed using advanced statistical and computational methods (64). The aims and final outcomes of this analysis can be diverse. For example, the analysis can be used to classify the patients in different groups according to their expression profiles and verify whether this correlates with the progression status of the investigated disease. In such an approach, one can obtain disease-associated genetic signatures, a group of genes whose combined expression pattern is linked to the development of the disease. This procedure has been remarkably successful in oncology, and some of the genetic signatures have proved to have predictive abilities: they can predict the future progression of the disease in the relevant patients and are useful in designing prognostic tests (65). These techniques, although not yet completely established within respect toof inflammatory diseases, can be a promising approach to gain insights into the relevant genes involved.

The approach can also be used to map asthma-related signaling networks and help in designing functional experiments. Zou and coauthors combined microarrays with real-time PCR to identify and validate differentially expressed genes in a monkey model of allergic asthma (66). The authors tested approximately 40,000 RNAs and found 169 items with highly differentiated expression levels. These genes were grouped into five clusters with unique expression patterns, one containing asthma-related genes coding, e.g., eotaxin, PARC, MCP-1, and MCP-3. Park and collaborators performed transcriptional time courses in the lungs in a mouse model of asthma for $9 \mathrm{wk}$ after exposure to aerosolized ovalbumin (67). They found a total of 776 differentially expressed mRNAs. Among these, most of the immune-responsive mRNAs were found to be transiently upregulated in the early phase of the allergen treatment (within $1 \mathrm{wk}$ ), but not for a longer duration (up to $6 \mathrm{wk}$ ), thereby suggesting that homeostatic mechanisms act as potential regulators of an allergic inflammatory reaction in chronic asthma.

It is not only RNA that is accessible on a broader level; Gray and colleagues used sputum proteomics to identify potential biomarkers of inflammatory lung disease (68) including asthma, chronic obstructive pulmonary disease (COPD), cystic fibrosis, and bronchiectasis. Using surface-enhanced laser

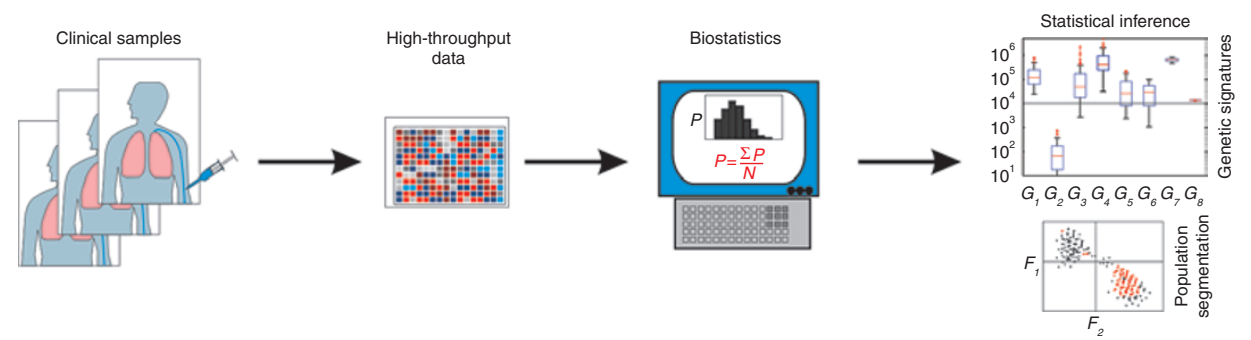

Figure 1. Workflow for "-omics" approaches. Clinical samples are obtained and processed to produce high-throughput data. The data are then analyzed using biostatistics. The aim of the analysis is to find correlations in the data, linking expression of biomolecules to the risk of developing a disease, its prognosis, or the efficacy of existing therapies. This approach can be used to find genetic signatures associated with the disease. These genetic signatures account for groups of genes whose combined expression pattern is linked to the development of the disease. 


\section{Review | sittkaetal.}

desorption/ionization time-of-flight to analyze the samples, the authors identified 105 potential biomarkers for asthma. Along similar lines, Zhao and coauthors investigated alterations in the global protein expression in the bronchoalveolar lavage fluid in a mouse model of allergic airway inflammation (69). The authors identified 28 proteins that were significantly altered in the experimental group as compared with the controls. The group of overexpressed proteins included lungkine, Ym1, Ym2, acidic mammalian chitinase, gob-5, and surfactant protein-D. Proteomics can also address protein modifications; Ghosh and collaborators evaluated the oxidation of proteins in an allergen-induced murine model of asthma (70). More than 30 different proteins were identified as nitration targets in response to an allergen challenge. Among them was the antioxidant enzyme catalase, the activity of which has been found to be downregulated by up to $50 \%$ in bronchoalveolar lavage fluid of humans with asthma. Further experiments showed increased oxidation in catalase isolated from asthmatic airway epithelial cells. These results pointed to the possibility that intense oxidative reactions contribute to the chronic inflammatory state found in asthmatic airways, consequent to the inactivation and loss of activity of catalase and other oxidativestress-related proteins. Data analysis is an essential approach in the study of asthma-associated pathophysiology; Jardim and coauthors used miRNA expression data in human airway cells from donors with asthma in order to identify asthmaassociated genes (71). After profiling miRNA expression of human bronchial epithelial cells, they found that the expression profiles of 66 miRNAs differed significantly between the two groups. Three of them were validated using real-time PCR data from an additional 16 asthma patients and 16 healthy subjects. A final set of functional assays was performed to confirm $A Q P 4$, a target of the highly downregulated miRNA-203 in asthmatic cells, as a novel asthma-associated gene.

It is possible to use omics data analysis to assess the global effect of anti-asthma drug administration. O'Neil and collaborators used quantitative proteomics on asthmatic bronchi to investigate the effects of inhaled glucocorticoid treatment (72). The authors carried out a double-blind treatment experiment with either placebo or budesonide $(800 \mu \mathrm{g}$ daily for $3 \mathrm{mo})$ on a cohort of 15 subjects, of which 12 were patients with asthma.
Endobronchial biopsies of the patients were obtained and analyzed using proteomics techniques. They found significant differences between treated and nontreated patients with respect to expression levels of proteins associated with pathways linked to acute phase response signaling, cell-to-cell communication, and tissue development.

\section{Data-Driven Mathematical Modeling of Biochemical Networks}

The mathematical modeling approach is focused more on establishing the biochemical mechanisms underlying a disease. In the past decade, experimental evidence has supported the idea that biological networks controlling cell function and tissue organization very often show nonlinear behavior. This originates in their structural complexity, which invalidates the use of direct intuition to understand their regulatory processes. Under these conditions, mathematical modeling of biochemical networks emerges as a methodology to dissect the function and regulation of biological networks (73). The method is an iterative process that involves: (i) the reconstruction of the relevant biochemical network using prior knowledge and the hypotheses under investigation; (ii) the translation of the network into a mathematical model; and (iii) the integration of the mathematical model with quantitative experimental data in iterative cycles, aiming to refine or reformulate the hypotheses (Figure 2). The outcome of the process is a more precise understanding of the structure, regulation, and function of the network, with the potential to help in the designing of more directed therapies.

The modeling of biochemical pathways involved in the regulation of the inflammatory response has been addressed in many papers during the past decade (74-78). Further, in the past few years several studies have adopted this approach to address some aspects of the molecular basis of asthma.

Hwang and coauthors constructed protein-protein interaction networks that represent proinflammatory and cellular proliferation signaling pathways in asthma, by extracting data from different resources including text mining of the OMIM database, microarrays from the GEO database, and proteinprotein interactions from the Human Protein Reference Database (79). The authors used the network to identify putative drug target genes for asthma.

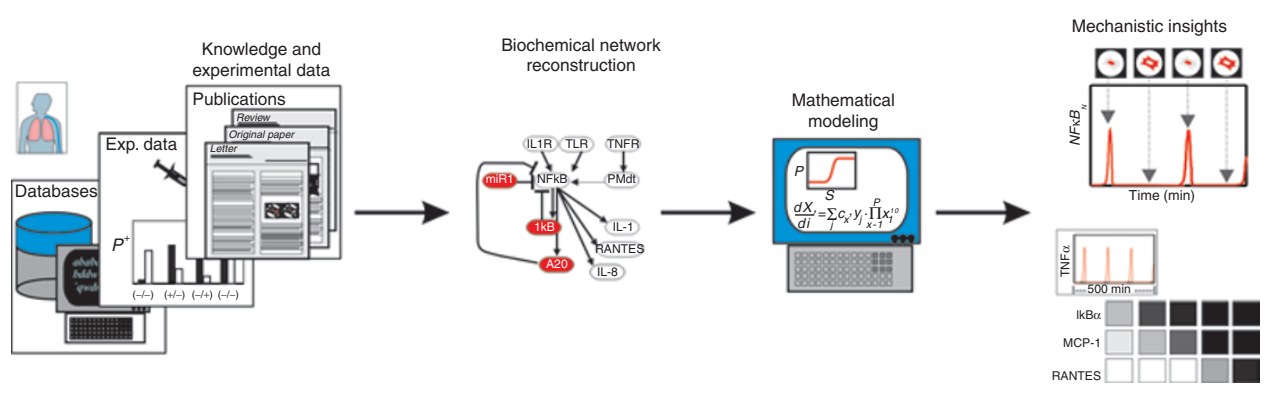

Figure 2. Workflow for a mathematical modeling approach. Published information, experimental data, and hypotheses are used to reconstruct the biochemical network that is relevant to a given disease. The network is translated into a mathematical model, which is characterized in iterative cycles, using quantitative experimental data on the temporal dynamics of its components under biological conditions that are relevant for the disease. This procedure can be used to prove hypotheses about the network regulation. In addition, the model may have predictive ability, thereby finding use in designing new experiments. 
Significant progress has also been made with respect to systems immunology. For instance, the IL-4-dependent activation of the important Th2-driving transcription factor GATA-3 has been modeled mathematically (80). The model implied that a bi-stable system is created by GATA-3 autoactivation, and that this can constitute a Th2 memory through a transient inductive signal. Subsequently, a model of differentiation of helper $\mathrm{T}$ cells was proposed, describing the expression of, and interactions between, the master regulators (i.e., transcription factors) that determine the phenotypic polarization toward Th1 or Th2 (81). Also, using elegant single-cell measurements and modeling, Podtschaske et al. showed that graded T-cell receptor signaling results in graded activation of the transcription factor nuclear factor $\kappa \mathrm{B}$, but a digital activation of transcription factor NFATc2 results in a switch that regulates the number of $\mathrm{T}$ lymphocytes actively participating in an immune response (82). In addition, cytokines such as IL-2, which have different effects on different populations of T lymphocytes, have also been studied by combined experimentation and modeling approaches specifically addressing the spatiotemporal complexity (75).

Mathematical modeling can also be a useful tool in biomedical research, ignoring the molecular details of the specific disease. For example, Frey and colleagues developed a mathematical model to investigate memory effects of the pathophysiology and pharmacodynamics of asthma (83). Using stochastic modeling and detrended fluctuation analysis, the authors investigated whether their methodology could be used to establish a correlation between past, current, and future lung function in patients. Toward this end, the authors computationally analyzed day-to-day data accounting for the fluctuations in lung function (i.e., peak expiratory flow) in patients with chronic asthma. They aimed to determine whether the future risk of asthma exacerbation could be predicted from fluctuations in peak expiratory flow, and how these results may be affected by bronchodilator therapy. Their method can also be used to predict the probability of future occurrences of asthma exacerbation, using current baseline fluctuation data of peak expiratory flow. A subsequent study by Frey's group indicated that, with regular use of short-acting $\beta_{2}$-agonists (but not long-acting ones), the fluctuation in lung function becomes more random and less predictable; according to the authors, this leads to increased risk of future occurrences of asthma exacerbation (84). There is also evidence to show that different types of asthma, such as cough-variant asthma and classical asthma, show different peak expiratory flow values. It is therefore possible to identify various asthma phenotypes using a simple parameter in Frey's model, conferring potentially important diagnostic value in the future (85).

\section{Multiscale Modeling of Asthma}

Mathematical models are also a useful strategy to integrate diverse sources of quantitative biological data. These data relate to different levels of organization in the body, from the regulation of intracellular biochemical networks to the structure and functioning of tissues and organs (86). This approach offers the potential to connect the genetic or epigenetic causes of a disease with their phenotypic effects at the organ level, using mathematical modeling. In line with this attempt, a multi-scale mathematical model was recently proposed to address airway hyperresponsiveness (87). The model is composed of different interconnected modules that are used to integrate experimental data for intracellular, cellular, tissue, and organ events that are relevant in asthma-related bronchoconstriction. For example, the model takes into account: (i) the kinetics of actin-myosin contractile protein production and depletion (intracellular event); (ii) the $\mathrm{Ca}^{2+}$-signaling mechanisms that regulate airway smooth muscle force production (cellular event); (iii) the interplay of mechanical forces that determine airway narrowing (tissue-level event); and (iv) the time-dependent distribution of airway smooth muscle contraction through the lung (whole-organ-level events). The authors claim that, when considered along with many other aspects of lung physiology, their model can be used to make predictions about bronchoconstriction, thereby helping to gain insights into airway hyperresponsiveness with a view to predicting potential therapies for asthma.

In a similar effort, Patel and coauthors developed a model to investigate lung deposition of inhaled drugs during asthma (88). Starting from a previously published model of the healthy human respiratory system (89), the authors developed a computational model that can simulate different levels of asthma severity, as well as various asthma-associated symptoms, including bronchoconstriction, inflammation, and mucus production. Further, the model allowed for the simulation of the administration of inhaled anti-asthma drugs. With the help of the model, various ventilation schemes (through different configurations of factors such as particle size and ventilatory parameters) were simulated and compared so as to investigate intersubject variability in breathing patterns after drug administration in asthma. This effort illustrated how computational models can be used to improve and personalize the therapy outcome in asthma patients.

\section{SYSTEMS MEDICINE APPROACH}

Within the past few decades, enormous scientific and technological progress has been made both in clinical medicine and biomedical research. However, there are two observations that are worrisomeying. First, despite all efforts by academia, the industry, government agencies, and even private foundations, there is still a tremendous unmet medical need with respect toas regards asthma treatment. Second, the gap between clinical medicine and basic research is getting wider in many countries. Even communication between different subdisciplines, e.g., immunologists, physiologists, and geneticists, is becoming more and more difficult. Theis second shortcoming might even hamper efforts to overcome the first one. Ironically, a third "kingdom"-mathematics-historically quite isolated from clinics and wet-lab research, might help to translate clinical problems in a valid way to basic science and the putative solutions back to the clinic to help patients.

The use of systems medicine in asthma could be a fruitful endeavor, given that the disease constitutes a huge socioeconomic burden, presents several clinical problems, challenges 


\section{Review | sittkaetal.}

scientific hypotheses, and is fully furnished with big clinical cohorts, multiple experimental models, and a tremendous amount of isolated scientific insights waiting for a big picture to emerge. One possible workflow of systems medicine in asthma is to integrate medical needs, patient cohorts undergoing comprehensive clinical phenotyping, and molecular omics data with a systems biology workbench, combining comprehensive bioinformatics and biostatistics, mathematical modeling, and experimental wet-lab research in animal, tissue, and cellular models. Figure 3 shows a workflow that will be established in the systems biology platform of the newly founded German Center for Lung Research, a huge translational endeavor of five German centers for pulmonary research funded by the federal government of Germany (90).

\section{Current Initiatives}

In recent years, the application of systems biology or systems medicine in respiratory medicine, especially asthma, has been attracting increasing interest from the scientific community and funding agencies in many countries. Some of the resulting scientific consortia are mentioned here. However, the list is not complete.

The Unbiased Biomarkers for the Prediction of Respiratory Disease Outcome Consortium (U-BIOPRED) is a European project involving scientists from universities, research institutes, the pharmaceutical industry and small companies, and part of the Innovative Medicines Initiative (91). It aims at a personalized management approach for patients with severe asthma by integrating omics data from invasively and noninvasively obtained patient material to define phenotypes with respect to therapeutic efficacy (7). It takes part in

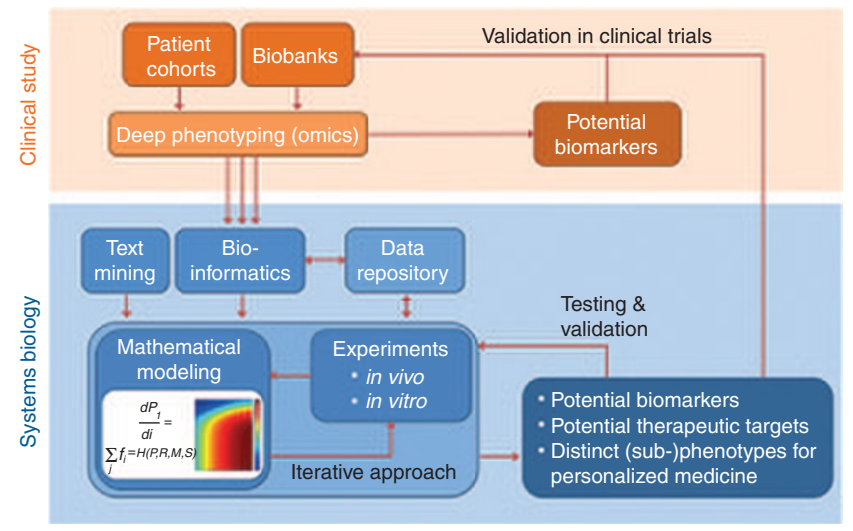

Figure 3. Systems medicine workflow. Here, the systems -omics and the molecular systems biology workflow are combined with data from patient cohorts undergoing comprehensive clinical phenotyping and biobanks in a circular-or iterative-approach. Pharmacologic, clinical, and molecular data were fed into a systems biology workbench through a comprehensive bioinformatics and biostatistics filter. In combination with existing data or information retrieved from literature, data repositories, etc., central network modules were extracted or defined. These modules can be translated into mathematical models with internal and external refinement and validation. Finally, predictions for potential biomarkers or therapeutic targets, together with the respective known or yet undescribed clinical subphenotypes of sensitive patients, can be translated back into clinical studies. the Airway Disease Predicting Outcomes Through Patient Specific Computational Modeling Consortium (AirPROM), a project funded by the European Union seventh framework program. AirPROM integrates expertise in physiology, radiology, image analysis, bioengineering, data harmonization, security and ethics, computational modeling, and systems biology to develop validated models that predict disease progression and response to treatment in asthma and COPD. Another project within the European Union seventh framework program is Synergy-COPD, which aims to develop a prediction tool to enable physicians to decide on the use of specific rehabilitation therapies for individual patients with COPD. As part of this, five previously validated mathematical models on oxygen uptake and usage from lung to mitochondria are to be integrated (92). Synergy-COPD is also part of the Virtual Physiological Human Network of excellence (VPH-NoE). Within the European Union, this project aims to integrate patient-specific computer models for personalized and predictive health care and tools for modeling and simulation of human physiology and disease-related processes. Different disease- or organ-centered exemplar projects exist. The project resembles the IUPS-Physiome project of the International Union of Physiological Sciences, which also contains a pulmonary disease branch, developing and coupling models of air flow, blood flow, soft tissue mechanics, gas exchange, heat and water balance, and inert gas mixing in anatomically relevant geometric meshes (93).

\section{CONCLUSION}

Recent developments contain perspectives for asthma prevention; on the other hand, they challenge our concepts of asthma development, pathophysiology, diagnostics, and therapy. Efforts aimed at a "systems medicine" approach to asthma treatment might be the only way to deal with the enormous amount of data arising from large asthma cohorts, complicated (including even prenatal) timelines, comprehensive clinical phenotyping, and technologic possibilities, such as nextgeneration sequencing and the use of genome-wide epigenetics, splice variants, RNA editing, microbiomes,. Although all these hold out promise of interesting times for scientists and for better patient care in the future, the integration of many professional disciplines, from in silico and in vitro to in vivo, will need enthusiasm and hard work from all sides and, last but not least, sustained funding, for this new discipline.

\section{ACKNOWLEDGMENTS}

We acknowledge excellent support from Marlene Sülberg in manuscript preparation and proofreading. We apologize to all colleagues whose excellent contributions to the field could not be included in this text due to space constraints.

\section{STATEMENT OF FINANCIAL SUPPORT}

Part of this work has been funded by the Federal Ministry of Education and Research (Forsys Lung-FKZ 0315256, and German Center for Lung Research-Systems Biology Platform) and the German Research Foundation (SFB/TR-84, IRTG 1673) to B.S., and the Hessen State Ministry of Higher Education, Research and the Arts (Loewe-UGMLC) to B.S. and A.S.

Disclosure: The authors declared no conflict of interest. 


\section{REFERENCES}

1. Busse WW, Lemanske RF Jr. Asthma. N Engl J Med 2001;344:350-62.

2. Eder W, Ege MJ, von Mutius E. The asthma epidemic. N Engl J Med 2006;355:2226-35.

3. Haldar P, Pavord ID, Shaw DE, et al. Cluster analysis and clinical asthma phenotypes. Am J Respir Crit Care Med 2008;178:218-24.

4. Barnes PJ. Immunology of asthma and chronic obstructive pulmonary disease. Nat Rev Immunol 2008;8:183-92.

5. Gonem S, Desai D, Siddiqui S, Brightling CC. Evidence for phenotypedriven treatment in asthmatic patients. Curr Opin Allergy Clin Immunol 2011;11:381-5.

6. McGrath KW, Icitovic N, Boushey HA, et al.; Asthma Clinical Research Network of the National Heart, Lung, and Blood Institute. A large subgroup of mild-to-moderate asthma is persistently noneosinophilic. Am J Respir Crit Care Med 2012;185:612-9.

7. Auffray C, Adcock IM, Chung KF, Djukanovic R, Pison C, Sterk PJ. An integrative systems biology approach to understanding pulmonary diseases. Chest 2010;137:1410-6.

8. Strachan DP. Hay fever, hygiene, and household size. BMJ 1989;299:1259-60.

9. Aberg N. Asthma and allergic rhinitis in Swedish conscripts. Clin Exp Allergy 1989;19:59-63.

10. Von Ehrenstein OS, Von Mutius E, Illi S, Baumann L, Böhm O, von Kries R. Reduced risk of hay fever and asthma among children of farmers. Clin Exp Allergy 2000;30:187-93.

11. Riedler J, Braun-Fahrländer C, Eder W, et al.; ALEX Study Team. Exposure to farming in early life and development of asthma and allergy: a cross-sectional survey. Lancet 2001;358:1129-33.

12. von Mutius E, Vercelli D. Farm living: effects on childhood asthma and allergy. Nat Rev Immunol 2010;10:861-8.

13. Brooks C, Pearce N, Douwes J. The hygiene hypothesis in allergy and asthma: an update. Curr Opin Allergy Clin Immunol 2013;13:70-7.

14. Chen Y, Blaser MJ. Helicobacter pylori colonization is inversely associated with childhood asthma. J Infect Dis 2008;198:553-60.

15. Braun-Fahrländer C, Riedler J, Herz U, et al.; Allergy and Endotoxin Study Team. Environmental exposure to endotoxin and its relation to asthma in school-age children. N Engl J Med 2002;347:869-77.

16. van Strien RT, Engel R, Holst O, et al.; ALEX Study Team. Microbial exposure of rural school children, as assessed by levels of $\mathrm{N}$-acetyl-muramic acid in mattress dust, and its association with respiratory health. J Allergy Clin Immunol 2004;113:860-7.

17. Ege MJ, Mayer M, Normand AC, et al.; GABRIELA Transregio 22 Study Group. Exposure to environmental microorganisms and childhood asthma. N Engl J Med 2011;364:701-9.

18. Genuneit J. Exposure to farming environments in childhood and asthma and wheeze in rural populations: a systematic review with meta-analysis. Pediatr Allergy Immunol 2012;23:509-18.

19. Macy E, Schatz M, Gibbons C, Zeiger R. The prevalence of reversible airflow obstruction and/or methacholine hyperreactivity in random adult asthma patients identified by administrative data. J Asthma 2005;42: 213-20.

20. Fuchs O, Genuneit J, Latzin P, et al.; GABRIELA Study Group. Farming environments and childhood atopy, wheeze, lung function, and exhaled nitric oxide. J Allergy Clin Immunol 2012;130:382-8.e6.

21. Omland $\varnothing$, Hjort C, Pedersen OF, Miller MR, Sigsgaard T. New-onset asthma and the effect of environment and occupation among farming and nonfarming rural subjects. J Allergy Clin Immunol 2011;128:761-5.

22. von Hertzen L, Hanski I, Haahtela T. Natural immunity. Biodiversity loss and inflammatory diseases are two global megatrends that might be related. EMBO Rep 2011;12:1089-93.

23. Hanski I, von Hertzen L, Fyhrquist N, et al. Environmental biodiversity, human microbiota, and allergy are interrelated. Proc Natl Acad Sci USA 2012;109:8334-9.

24. von Mutius E. A fascinating look at the world with a new microscope. J Allergy Clin Immunol 2012;129:1202-3.

25. Lim RH, Kobzik L. Maternal transmission of asthma risk. Am J Reprod Immunol 2009;61:1-10.
26. McKeever TM, Lewis SA, Smith C, Hubbard R. The importance of prenatal exposures on the development of allergic disease: a birth cohort study using the West Midlands General Practice Database. Am J Respir Crit Care Med 2002;166:827-32.

27. Ege MJ, Bieli C, Frei R, et al.; Parsifal Study team. Prenatal farm exposure is related to the expression of receptors of the innate immunity and to atopic sensitization in school-age children. J Allergy Clin Immunol 2006;117:817-23.

28. Douwes J, Cheng S, Travier N, et al. Farm exposure in utero may protect against asthma, hay fever and eczema. Eur Respir J 2008;32:603-11.

29. Mold JE, Michaëlsson J, Burt TD, et al. Maternal alloantigens promote the development of tolerogenic fetal regulatory $\mathrm{T}$ cells in utero. Science 2008;322:1562-5.

30. Schaub B, Liu J, Höppler S, et al. Maternal farm exposure modulates neonatal immune mechanisms through regulatory T cells. J Allergy Clin Immunol 2009;123:774-82.e5.

31. Pfefferle PI, Büchele G, Blümer N, et al.; PASTURE Study Group. Cord blood cytokines are modulated by maternal farming activities and consumption of farm dairy products during pregnancy: the PASTURE Study. J Allergy Clin Immunol 2010;125:108-15.e1-3.

32. Macaubas C, de Klerk NH, Holt BJ, et al. Association between antenatal cytokine production and the development of atopy and asthma at age 6 years. Lancet 2003;362:1192-7.

33. Blümer N, Herz U, Wegmann M, Renz H. Prenatal lipopolysaccharideexposure prevents allergic sensitization and airway inflammation, but not airway responsiveness in a murine model of experimental asthma. Clin Exp Allergy 2005;35:397-402.

34. Conrad ML, Ferstl R, Teich R, et al. Maternal TLR signaling is required for prenatal asthma protection by the nonpathogenic microbe Acinetobacter lwoffii F78. J Exp Med 2009;206:2869-77.

35. Brand S, Teich R, Dicke T, et al. Epigenetic regulation in murine offspring as a novel mechanism for transmaternal asthma protection induced by microbes. J Allergy Clin Immunol 2011;128:618-25.e1-7.

36. McCallister JW. Reslizumab and eosinophilic asthma: one step closer to phenotype-directed therapy? Am J Respir Crit Care Med 2011;184:10967.

37. Martinez FD, Chinchilli VM, Morgan WJ, et al. Use of beclomethasone dipropionate as rescue treatment for children with mild persistent asthma (TREXA): a randomised, double-blind, placebo-controlled trial. Lancet 2011;377:650-7.

38. Zeiger RS, Mauger D, Bacharier LB, et al.; CARE Network of the National Heart, Lung, and Blood Institute. Daily or intermittent budesonide in preschool children with recurrent wheezing. N Engl J Med 2011;365:19902001.

39. Moore WC, Meyers DA, Wenzel SE, et al.; National Heart, Lung, and Blood Institute's Severe Asthma Research Program. Identification of asthma phenotypes using cluster analysis in the Severe Asthma Research Program. Am J Respir Crit Care Med 2010;181:315-23.

40. Siroux V, Basagaña X, Boudier A, et al. Identifying adult asthma phenotypes using a clustering approach. Eur Respir J 2011;38:310-7.

41. Wenzel SE, Vitari CA, Shende M, Strollo DC, Larkin A, Yousem SA. Asthmatic granulomatosis: a novel disease with asthmatic and granulomatous features. Am J Respir Crit Care Med 2012;186:501-7.

42. Fleming L, Tsartsali L, Wilson N, Regamey N, Bush A. Sputum inflammatory phenotypes are not stable in children with asthma. Thorax 2012;67:675-81.

43. Wenzel SE. Asthma phenotypes: the evolution from clinical to molecular approaches. Nat Med 2012;18:716-25.

44. Tantisira KG, Lasky-Su J, Harada M, et al. Genomewide association between GLCCI1 and response to glucocorticoid therapy in asthma. N Engl J Med 2011;365:1173-83.

45. Wechsler ME, Castro M, Lehman E, et al.; NHLBI Asthma Clinical Research Network. Impact of race on asthma treatment failures in the asthma clinical research network. Am J Respir Crit Care Med 2011;184:1247-53.

46. Weatherall M, Wijesinghe M, Perrin K, Harwood M, Beasley R. Meta-analysis of the risk of mortality with salmeterol and the effect of concomitant inhaled corticosteroid therapy. Thorax 2010;65:39-43. 
47. Bleecker ER, Nelson HS, Kraft M, et al. Beta2-receptor polymorphisms in patients receiving salmeterol with or without fluticasone propionate. Am J Respir Crit Care Med 2010;181:676-87.

48. Iwamoto H, Yokoyama A, Shiota N, et al. Tiotropium bromide is effective for severe asthma with noneosinophilic phenotype. Eur Respir J 2008;31:1379-80.

49. Simpson JL, Powell H, Boyle MJ, Scott RJ, Gibson PG. Clarithromycin targets neutrophilic airway inflammation in refractory asthma. Am J Respir Crit Care Med 2008;177:148-55.

50 A plea to abandon asthma as a disease concept. Lancet 2006;368:705.

51. Anderson GP. Endotyping asthma: new insights into key pathogenic mechanisms in a complex, heterogeneous disease. Lancet 2008;372:1107-19.

52. Busse WW, Morgan WJ, Gergen PJ, et al. Randomized trial of omalizumab (anti-IgE) for asthma in inner-city children. N Engl J Med 2011;364:1005-15.

53. Leckie MJ, ten Brinke A, Khan J, et al. Effects of an interleukin-5 blocking monoclonal antibody on eosinophils, airway hyper-responsiveness, and the late asthmatic response. Lancet 2000;356:2144-8.

54. Haldar P, Brightling CE, Hargadon B, et al. Mepolizumab and exacerbations of refractory eosinophilic asthma. N Engl J Med 2009;360:973-84.

55. Nair P, Pizzichini MM, Kjarsgaard $M$, et al. Mepolizumab for prednisone-dependent asthma with sputum eosinophilia. N Engl J Med 2009;360:985-93.

56. Castro M, Mathur S, Hargreave F, et al.; Res-5-0010 Study Group. Reslizumab for poorly controlled, eosinophilic asthma: a randomized, placebocontrolled study. Am J Respir Crit Care Med 2011;184:1125-32.

57. Borish LC, Nelson HS, Lanz MJ, et al. Interleukin-4 receptor in moderate atopic asthma. A phase I/II randomized, placebo-controlled trial. Am J Respir Crit Care Med 1999;160:1816-23.

58. Maes T, Joos GF, Brusselle GG. Targeting interleukin-4 in asthma: lost in translation? Am J Respir Cell Mol Biol 2012;47:261-70.

59. Corren J, Lemanske RF, Hanania NA, et al. Lebrikizumab treatment in adults with asthma. N Engl J Med 2011;365:1088-98.

60. Berry MA, Hargadon B, Shelley M, et al. Evidence of a role of tumor necrosis factor alpha in refractory asthma. N Engl J Med 2006;354:697-708.

61. Holgate ST, Noonan M, Chanez P, et al. Efficacy and safety of etanercept in moderate-to-severe asthma: a randomised, controlled trial. Eur Respir J 2011;37:1352-9.

62. Mestas J, Hughes CC. Of mice and not men: differences between mouse and human immunology. J Immunol 2004;172:2731-8.

63. Wenzel S, Holgate ST. The mouse trap: It still yields few answers in asthma. Am J Respir Crit Care Med 2006;174:1173-6; discussion 1176-8.

64. Quackenbush J. Computational approaches to analysis of DNA microarray data. Yearb Med Inform 2006;91-103.

65. van ' $t$ Veer LJ, Dai H, van de Vijver MJ, et al. Gene expression profiling predicts clinical outcome of breast cancer. Nature 2002;415:530-6.

66. Zou J, Young S, Zhu F, et al. Microarray profile of differentially expressed genes in a monkey model of allergic asthma. Genome Biol 2002;3:research0020.

67. Park SG, Choi JW, Kim Hj, et al. Genome-wide profiling of antigen-induced time course expression using murine models for acute and chronic asthma. Int Arch Allergy Immunol 2008;146:44-56.

68. Gray RD, MacGregor G, Noble D, et al. Sputum proteomics in inflammatory and suppurative respiratory diseases. Am J Respir Crit Care Med 2008; 178:444-52.

69. Zhao J, Zhu H, Wong CH, Leung KY, Wong WS. Increased lungkine and chitinase levels in allergic airway inflammation: a proteomics approach. Proteomics 2005;5:2799-807.

70. Ghosh S, Janocha AJ, Aronica MA, et al. Nitrotyrosine proteome survey in asthma identifies oxidative mechanism of catalase inactivation. J Immunol 2006;176:5587-97.

71. Jardim MJ, Dailey L, Silbajoris R, Diaz-Sanchez D. Distinct microRNA expression in human airway cells of asthmatic donors identifies a novel asthma-associated gene. Am J Respir Cell Mol Biol 2012;47:536-42.
72. O'Neil SE, Sitkauskiene B, Babusyte A, et al. Network analysis of quantitative proteomics on asthmatic bronchi: effects of inhaled glucocorticoid treatment. Respir Res 2011;12:124.

73. Vera J, Wolkenhauer O. A system biology approach to understand functional activity of cell communication systems. Methods Cell Biol 2008;90:399-415.

74. Ashall L, Horton CA, Nelson DE, et al. Pulsatile stimulation determines timing and specificity of NF-kappaB-dependent transcription. Science 2009;324:242-6.

75. Busse D, de la Rosa M, Hobiger K, et al. Competing feedback loops shape IL-2 signaling between helper and regulatory T lymphocytes in cellular microenvironments. Proc Natl Acad Sci USA 2010;107:3058-63.

76. Hoffmann A, Levchenko A, Scott ML, Baltimore D. The IkappaB-NFkappaB signaling module: temporal control and selective gene activation. Science 2002;298:1241-5.

77. Nelson DE, Ihekwaba AE, Elliott M, et al. Oscillations in NF-kappaB sig naling control the dynamics of gene expression. Science 2004;306:704-8.

78. Nikolov S, Vera J, Rath O, Kolch W, Wolkenhauer O. Role of inhibitory proteins as modulators of oscillations in NFB signalling. IET Syst Biol 2009;3:59-76.

79. Hwang S, Son SW, Kim SC, Kim YJ, Jeong H, Lee D. A protein interaction network associated with asthma. J Theor Biol 2008;252:722-31.

80. Höfer T, Nathansen H, Löhning M, Radbruch A, Heinrich R. GATA-3 transcriptional imprinting in Th2 lymphocytes: a mathematical model. Proc Natl Acad Sci USA 2002;99:9364-8.

81. van den Ham HJ, de Boer RJ. From the two-dimensional Th1 and Th2 phenotypes to high-dimensional models for gene regulation. Int Immunol 2008;20:1269-77.

82. Podtschaske M, Benary U, Zwinger S, Höfer T, Radbruch A, Baumgrass R. Digital NFATc2 activation per cell transforms graded T cell receptor activation into an all-or-none IL-2 expression. PLoS ONE 2007;2:e935.

83. Frey U, Brodbeck T, Majumdar A, et al. Risk of severe asthma episodes predicted from fluctuation analysis of airway function. Nature 2005;438:667-70

84. Thamrin C, Stern G, Strippoli MP, et al. Fluctuation analysis of lung function as a predictor of long-term response to beta2-agonists. Eur Respir J 2009;33:486-93.

85. Frey U. Asthma as a nonlinear complex dynamic system: a novel approach to understand the temporal behaviour of chronic asthma and its response to $\beta$-agonists. Eur Respir Rev 2008;17:67-9.

86. Hunter P, Smith N, Fernandez J, Tawhai M. Integration from proteins to organs: the IUPS Physiome Project. Mech Ageing Dev 2005;126:187-92.

87. Lauzon AM, Bates JH, Donovan G, Tawhai M, Sneyd J, Sanderson MJ. A multi-scale approach to airway hyperresponsiveness: from molecule to organ. Front Physiol 2012;3:191.

88. Patel B, Gauvin R, Absar S, et al. Computational and bioengineered lungs as alternatives to whole animal, isolated organ, and cell-based lung models. Am J Physiol Lung Cell Mol Physiol 2012;303:L733-47.

89. Martonen T, Fleming J, Schroeter J, Conway J, Hwang D. In silico modeling of asthma. Adv Drug Deliv Rev 2003;55:829-49.

90. Seeger W, Welte T, Eickelberg O, et al. The German centre for lung research - translational research for the prevention, diagnosis and treatment of respiratory diseases. Pneumologie 2012;66:464-9.

91. Bel EH, Sousa A, Fleming L, et al.; Unbiased Biomarkers for the Prediction of Respiratory Disease Outcome (U-BIOPRED) Consortium, Consensus Generation. Diagnosis and definition of severe refractory asthma: an international consensus statement from the Innovative Medicine Initiative (IMI). Thorax 2011;66:910-7.

92. Maier D, Kalus W, Wolff M, et al. Knowledge management for systems biology a general and visually driven framework applied to translational medicine. BMC Syst Biol 2011;5:38.

93. Politi AZ, Donovan GM, Tawhai MH, et al. A multiscale, spatially distributed model of asthmatic airway hyper-responsiveness. J Theor Biol 2010;266:614-24. 